\title{
Image De-blurring and Supper-Resolution by Adaptive Sparse Domain Selection and Regularization
}

\author{
Monika Banafar \\ M.Tech Scholar \\ $\mathrm{EC}(\mathrm{DC})$ \\ NIRT,RGPV, Bhopal, India
}

\author{
Deepak Kourav \\ Assi. Professor \\ $\mathrm{EC}(\mathrm{DC})$ \\ NIRT,RGPV, Bhopal, India
}

\begin{abstract}
The method of total variation is used as a significant and competent image prior model in the regularization based area of image processing. However, as the model showing total variation supports a piecewise steady explanation, this process is classify below high intensity noise in the level areas of the picture is often poor, and a small number of pseudo edges are produced. Under this work we build up a spatially adaptive total variation model. Initially, we extract the spatial data based on each and every pixel, then two filtering process are combined to control the collision of pseudo edges. It also includes, the spatial information weight is build and classified with k-means clustering, and the cluster controls the center value of regularization strength in every region. The tentative results, of both simulated as well as genuine datasets, exhibit that the projected methodology can effectively diminish the pseudo edges formed by the total variation regularization in the flat areas, and maintain the limited smoothness of the HR images. The proposed region based spatial information adaptive variation model can effectively reduce the cause of noise on the spatial data extraction and maintain strength with changes in the noise intensity in the SR process as compare to traditional pixel based spatial information adaptive methodology.
\end{abstract}

\section{Keywords}

Total variation, regional spatially adaptive, Super resolution, High resolution, Majorization-minimization.

\section{INTRODUCTION}

HIGH-RESOLUTION (HR) symbolism plays a key element in various dissimilar ranges of use, for example restorative imaging, video surveillance and remote sensing.[1] On the other hand, in light of the fact that there are various constraints with both the hypothetical and practical viewpoints, for example, the sensor resolution and high cost, among different things, it's very hard to acquire a HR image than a low-resolution image. Thus, scientists have reconnoitered approaches to get a HR image from the image processing phases and recently the super-resolution (SR) technology which yields a High resolution image from single or multiframe Low resolution has been proposed.[5] Our exploration is principally centered on the multiframe image $\mathrm{SR}$ issue, the procedure to reconstruct a HR image from the sequence of LR image.[5]

\subsection{Super-Resolution}

In an image, image resolution is defined as the tiniest visible or measurable detail in a visual presentation that refers the spacing of pixels.[1] As the number of pixel is greater the spatial resolution of an image is also higher. For imaging applications, high resolution images are essential. By decreasing the pixel size we can get a high resolution image .[1]It can be reduced to a certain limit because , if the pixel size is reduced the availability of light is also reduced . Due to which shot noise is also arises and the image gets degraded. To overcome with this problem we have to reduce the pixel size up to a certain limit. Super-resolution imaging (SR) is a technique which increases the resolution of various image system .Some SR techniques are optical SR and geometrical SR techniques. In optical SR technique the diffraction limit of the system is transcended and in geometrical SR technique the resolution of the digital imaging sensor is enhanced.[3] Both the techniques are useful in general image processing and super resolution microscopy.

\subsection{Total Variation De-noising (TVD)}

Under signal processing field total variation de-noising, is also recognized as total variation regularization.[7]This process is used in digital image processing which helps in removal of noise. It is based on the standard that signals with extreme and perhaps spurious detail has high variation. The integral of the gradient of the signal is high. According to this principle, reduction of the total variation of the signal tends to a close match with the original signal; it prevents the important detail like edges and removes unwanted detail.
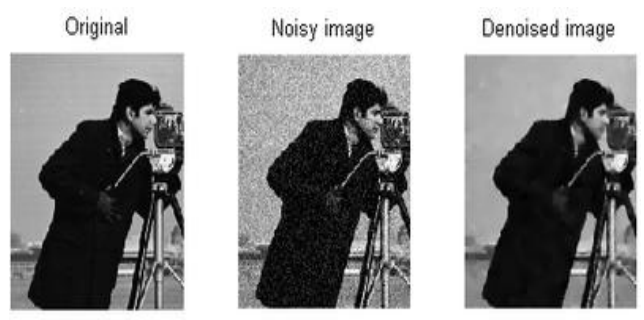

Figure:1 Example of TVD

The concept was initiated by Rudin et al. in 1992 .

This technique of noise removal has advantages over the simple techniques like linear smoothing which is also known as median filtering.[6] This technique reduces noise as well as smooth away the edges to a large degree.[6] This technique is extremely helpful and at the same time preserving edges as well as smoothing away noise in flat region, still at small signal-to-noise ratios.

\subsection{Total Variation}

This measures the changes between the signal values. Specifically, in total variation of an N-point signal $\mathrm{x}(\mathrm{n}), 1 \leq \mathrm{n}$ $\leq \mathrm{N}$ is defined as

$$
T V(x)=\sum_{n=2}^{N}|x(n)-x(n-1)|
$$


The total variation of $\mathrm{x}$ can written as

$\mathrm{TV}(\mathrm{x})=\left\|\mathrm{D}_{\mathrm{x}}\right\|_{1}$

Where $\|\cdot\|$ is the $l_{1}$ norm and

\section{PROPOSED METHOD}

Here we have created a simple spatially adaptive total variation model. First of all the spatial data is taken out from each and every pixel, for smoothing the impact of pseudo edges we add two filters.[2] Then, we can construct and classify the spatial information weight with the help of kmeans clustering, and the cluster center value controls regularization strength in every region.[2] The tentative results of simulated and genuine datasets, demonstrate that the proposed methodology can effectively lessen the pseudo edges of the total variation regularization in the flat areas, and keep up the partial smoothness of the HR images.[6] More significantly with the comparison of the usual pixel based spatial data adaptive method, the method based on proposed region can do better i.e., in the super resolution process it helps in avoiding the effect of noise and maintains the robustness with changes in the intensity of noise.[10] First of all we have to take an original image and after that apply down sampling algorithm to design so that we can extract spatial information. After that we add noise to the sampled data such as Gaussian noise and then we can filter the data using clustering algorithm to calculate $\mathrm{K}$ means clustering of the image which is taken as a sample. For de-noising the data samples apply MM algorithm .Lastly by using RSATV algorithm .We can get high resolution image as the output.

\section{RESULT}

In this work, we create a provincial spatially adaptive total variation model. At first, the spatial data is extracted from every pixel, and at that point two filters are added to smother the impact of pseudo edges. After then, the spatial information weight is constructed and classify by k-means clustering, the regularization strength in every region is controlled by the cluster center value. The investigative results of simulated as well as genuine datasets, demonstrate that the proposed methodology can effectively lessen the pseudo edges of the total variation regularization in the flat areas, and keep up the partial smoothness of the HR images. More importantly if we compare it with the traditional pixel based spatial data adaptive method, the proposed region based can perform better i.e., can avoid the effect of noise on the spatial information extraction and maintains the robustness with changes in intensity of noise in the super resolution process. As you see below the given figures, firstly we took an original image and then apply down sampling algorithm to design and then we extract spatial information of the sampled data and then we add noise to the sampled data like Gaussian noise and then filtering the data with the help of clustering algorithm. Calculate K means clustering of an image sample and apply $\mathrm{mm}$ algorithm for the noisy image to de-noise of data sample and then apply RSATV algorithm get high resolution image output as show on figure:

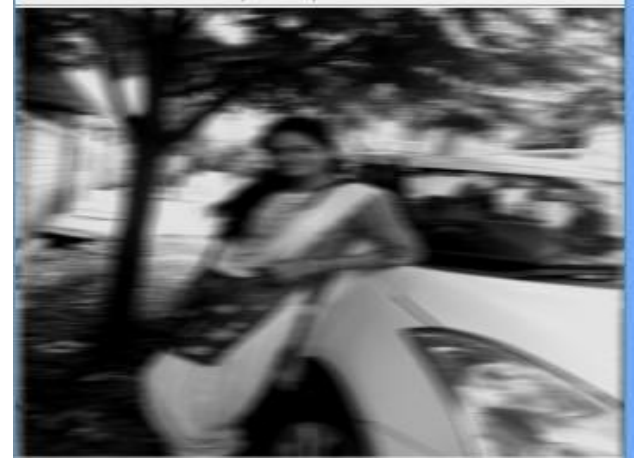

Figure 2: Input Image

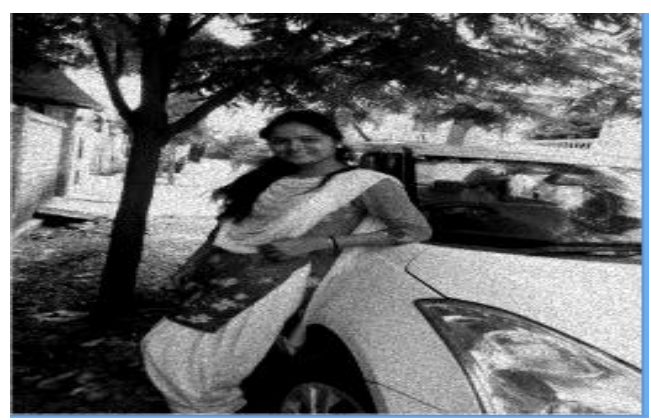

Figure 3: Image After Applying Down Sampling

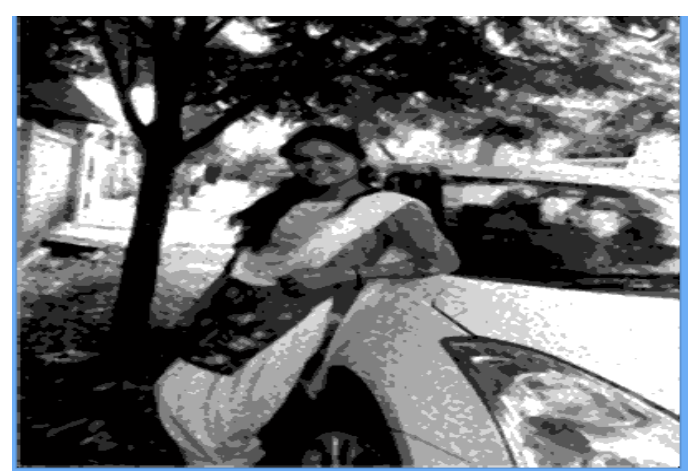

Figure 4: Image Corrupted With Noise

Table: 1 Comparison PSNR value between base paper and Proposed

\begin{tabular}{|c|c|c|c|}
\hline Noise variance & Value & $\begin{array}{c}\text { Previous } \\
\text { technique } \\
\text { RSATV }\end{array}$ & $\begin{array}{c}\text { New } \\
\text { technique } \\
\text { RSATV + } \\
\text { TVD }\end{array}$ \\
\hline \multirow{2}{*}{8} & PSNR & 32.14 & 40.76 \\
\hline \multirow{2}{*}{14} & SSIM & 0.964 & 0.96 \\
\cline { 2 - 4 } & PSNR & 28.14 & 28.90 \\
\cline { 2 - 4 } & SSIM & 0.918 & 0.92 \\
\hline \multirow{2}{*}{18} & PSNR & 26.46 & 26.9 \\
\cline { 2 - 5 } & SSIM & 0.897 & 0.9 \\
\hline
\end{tabular}




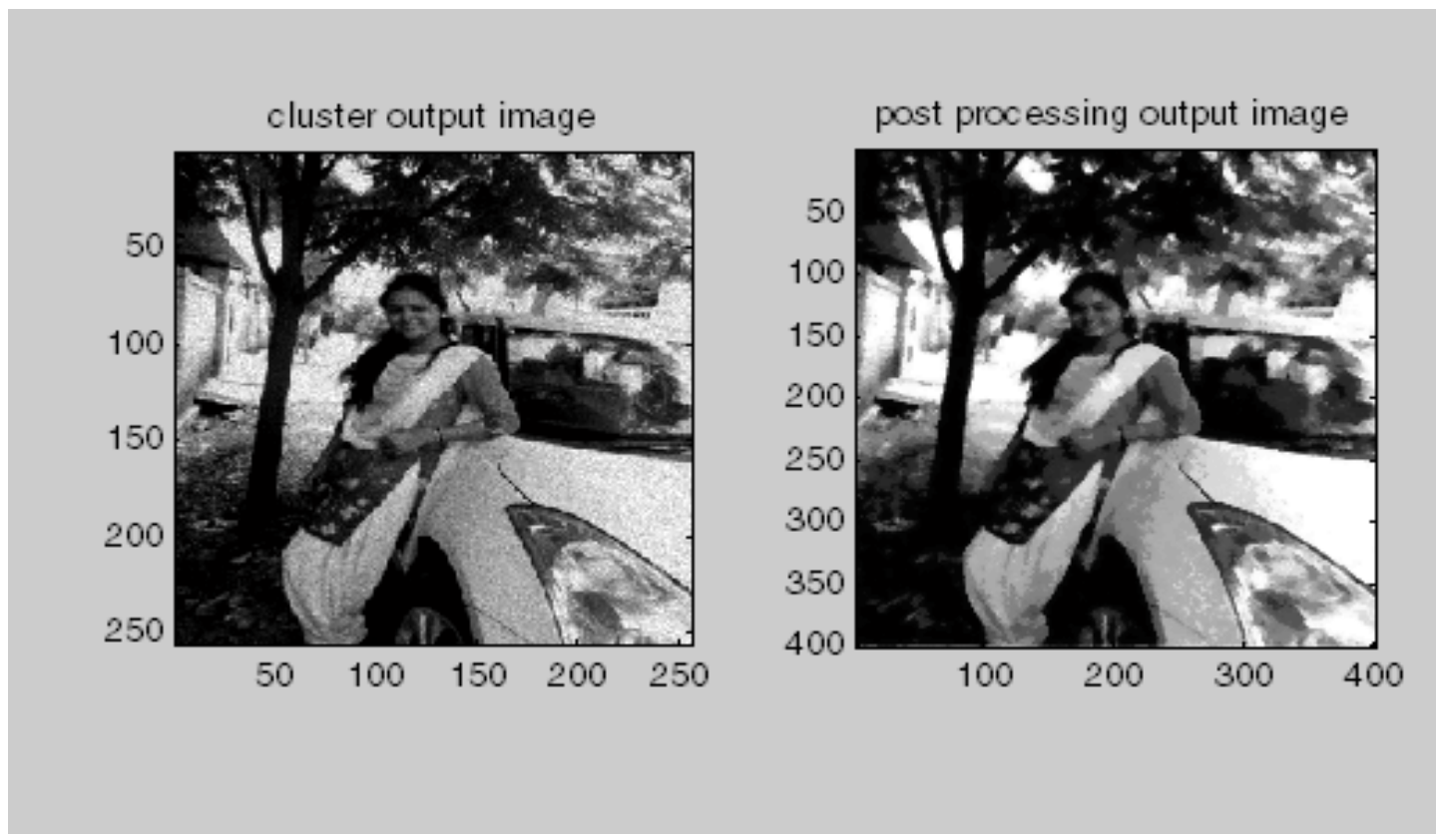

Figure 5: Input Image Comparing With Output Post Processed Image

\section{CONCLUSION}

The conventional spatially adaptive total variation model has the inadequacy of being weak to noise, and it performs inadequately in high noise intensity conditions. To beat this, in this paper, we propose a Regional spatially adaptive (RSATV) super-resolution calculation with spatial data filtering and clustering. The spatial data is initially extricated for every pixel, and after that the spatial data filtering procedure and spatial weight clustering methodology are included. With the help of these methods regularization quality of the total variation model is balanced for every area with distinctive spatial information, instead of for every pixel, as in the conventional spatially adaptive TV model. The real data information examinations introduced in above Section demonstrate that the proposed RSATV model can better supress the noise, without losing the edge and flat region data.

\section{REFERENCES}

[1] H. Greenspan, "Super-resolution in medical imaging," Comput. J,ol. 52, no. 1, pp. 43-63, Jan. 2009.

[2] X. Huang, L. Zhang, and P. Li, "Classification and extraction of spatial features in urban areas using highresolution multispectral imagery,"IEEE Trans. Geosci. Remote Sens. Lett., vol. 4, no. 2, pp. 260-264,Apr. 2007.

[3] X. Huang and L. Zhang, "An adaptive mean-shift analysis approach for object extraction and classification from urban hyper spectral imagery,"IEEE Trans. Geosci. Remote Sens., vol. 46, no. 12, pp. 4173-4185,Dec. 2008.

[4] L. Zhang, H. Zhang, H. Sheen, and P. Li, "A superresolution reconstruction algorithm for surveillance images," Signal Process., vol. 90, no. 3, pp. 848-859, 2010.
[5] R. Tsai and T. Huang, "Multiple frame image restoration and registration," Adv. Comput.Vis. Image Process., vol. 1, no. 2, pp. 317-339, 1984.

[6] S. Kim, N. Bose, and H. Valenzuela, "Recursive reconstruction of high resolution image from noisy under sampled multi frames," IEEE Trans. Acoust., Speech, Signal Process., vol. 38, no. 6, pp. 1013-1027, Jun. 1990.

[7] S. Kim and W. Su, "Recursive high-resolution reconstruction of blurred multiframe images," IEEE Trans. Image Process., vol. 2, no. 4, pp. 534-539, Oct. 1993.

[8] H. Ur and D. Gross, "Improved resolution from sub-pixel shifted pictures," Comput. Vis. Graph., Graph. Models Image Process., vol. 54, no. 2, pp. 181-186, 1992.

[9] M. Alam, J. Bognar, R. Hardie, and B. Yasuda, "Infrared image registration and high-resolution reconstruction using multiple translationally shifted aliased video frames," IEEE Trans. Instrum. Meas., vol. 49, no. 5, pp. 915-923, Oct. 2000.

[10] B. Tom and A. Katsaggelos, "Reconstruction of a highresolution image by simultaneous registration, restoration, and interpolation of low resolution images," in Proc. IEEE Int. Conf. Image Process., vol. 2. Washington, DC, USA, 1995, pp. 539-542.

[11] R. Schultz and R. Stevenson, "Extraction of highresolution frames from video sequences," IEEE Trans Image Process., vol. 5, no. 6, pp. 996-1011, Jun. 1996.

[12] S. Belekos, N. Galatsanos, and A. Katsaggelos, "Maximum a posteriori video super-resolution using a new multichannel image prior," IEEE Trans. Image Process., vol. 19, no. 6, pp. 1451-1464, Jun. 2010. 